\title{
Trombose de veia central da retina em paciente usuária de interferon e ribavirina: relato de caso
}

\author{
Central vein occlusion in a patient using interferon and riba virin:case report
}

\author{
John Helal Jr. ${ }^{1}$ \\ LeandroCabral Zacharias ${ }^{2}$ \\ Luciana Malta de Alencar ${ }^{3}$
}

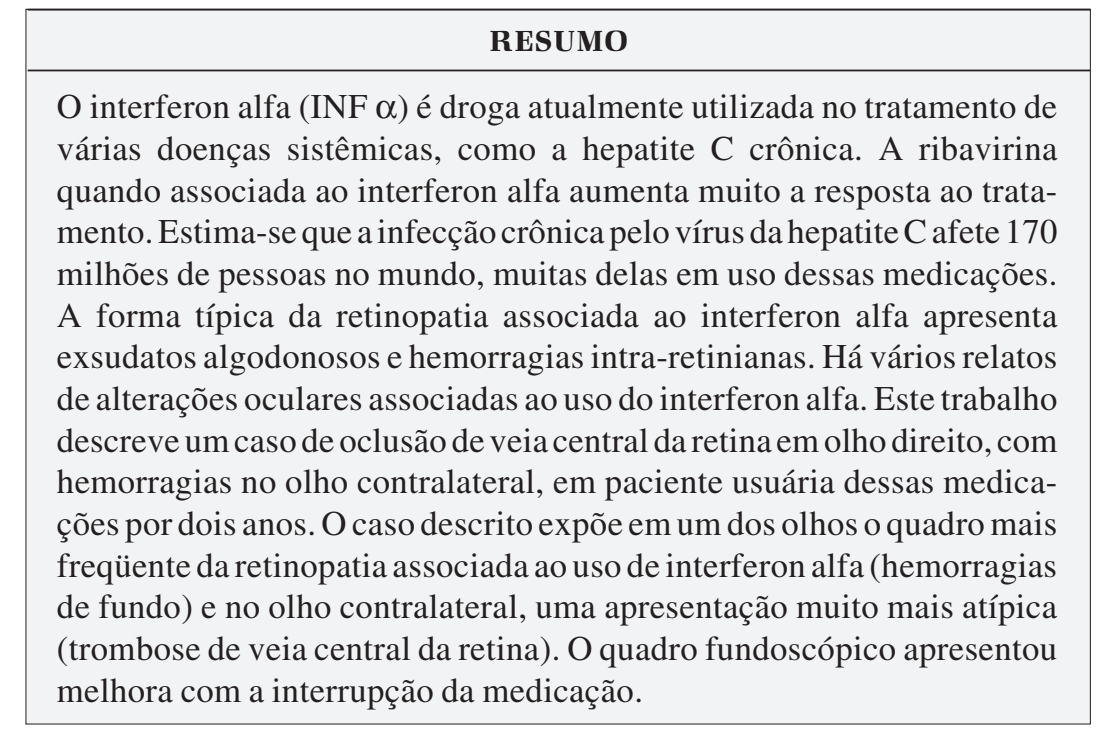

Descritores: Hepatite C; Ribavirina/efeitos adversos; Interferon/efeitos adversos; Retinopatia; Oclusão da veia retiniana; Relato de casos [tipo de publicação]

\section{INTRODUÇÃO}

A infecção crônica pelo vírus da hepatite $\mathrm{C}$ afeta cerca de 4 milhões de pessoas nos Estados Unidos e aproximadamente 170 milhões em todo o mundo ${ }^{(1-2)}$. Somente 15 a $20 \%$ destes pacientes apresentam resposta sustentada à terapia com interferon alfa - INF $\alpha^{(3-4)}$, mas a combinação da terapia com ribavirina aumenta significativamente esse índice ${ }^{(5-6)}$. O INF $\alpha$, um agrupamento complexo de proteínas, com atividades antivirais, antiproliferativas e imunorregulatórias, é uma droga atualmente utilizada não só no tratamento da hepatite C crônica, mas também de condições sistêmicas, como o sarcoma de Kaposi, os hemangiomas, os melanomas cutâneos e o carcinoma renal metastático ${ }^{(7)}$. A ribavirina, por sua vez, é um nucleosídio sintético, análogo à guanidina, com propriedades anti-retrovirais contra uma série de DNA e RNA vírus $^{(8)}$, e também com atividade imunorregulatória ${ }^{(9)}$.

A terapia com INFo é associada a vários efeitos colaterais sistêmicos, como fadiga, mal-estar, perda de peso, depressão, náusea, anorexia, diarréia, neutropenia e trombocitopenia ${ }^{(10-12)}$. Em 1993, Guyer e colaboradores descreveram uma forma de retinopatia associada à terapia com interferon, marcada pela presença de exsudatos algodonosos e hemorragias intraretinianas $^{(13)}$. Desde então, vários relatos demonstraram alterações oculares associadas ao uso desta droga.

A retinopatia típica inclui exsudatos algodonosos e hemorragias retinia- 
nas localizadas preferencialmente no fundo, próximos ao nervo óptico. Esse quadro é normalmente assintomático, reversível e associado a uma boa acuidade visual ${ }^{(14-15)}$. Alguns pacientes, entretanto, podem desenvolver isquemia por nãoperfusão dos capilares retinianos, edema retiniano, extravasamento vascular, anormalidades microvasculares e oclusões, com dano retiniano severo e conseqüente baixa de acuidade visual $^{(13)}$. Quadros oculares atípicos incluem hemorragia subconjuntival, hemorragia vítrea, glaucoma neovascular, edema do nervo óptico e paralisias oculomotoras ${ }^{(13-14,16)}$; entretanto oclusões de ramo arterial, de ramo venoso ou da veia central da retina, embora bastante infreqüentes, já foram associadas ao uso da terapia com INF $\alpha^{(14,17)}$.

A despeito dos efeitos colaterais com o uso sistêmico de ribavirina incluírem somente lacrimejamento e conjuntivite, essa medicação pode contribuir para a retinopatia em razão de sua ação sinergística, quando utilizada em combinação com o interferon $^{(5,14)}$.

Relata-se aqui um caso de oclusão de veia central da retina, associado a hemorragias no olho contralateral em paciente portadora de hepatite C usuária de INF $\alpha$ e ribavirina.

\section{RELATO DO CASO}

LHL, 64 anos, sexo feminino, natural de São José dos Campos - SP, economista. A paciente é, há mais de 10 anos, portadora de glaucoma primário de ângulo aberto em ambos os olhos e, desde então, vem sendo acompanhada por um especialista. Há dois anos, após diagnóstico de hepatite $\mathrm{C}$, passou a ser tratada com INF $\alpha$ subcutâneo e ribavirina via oral por seis meses. O tratamento foi interrompido durante dez meses e há um ano voltou a receber as mesmas medicações. Não havia história anterior de diabetes mellitus, hipertensão arterial sistêmica ou outras comorbidades.

No exame prévio, apresentou acuidade visual corrigida de

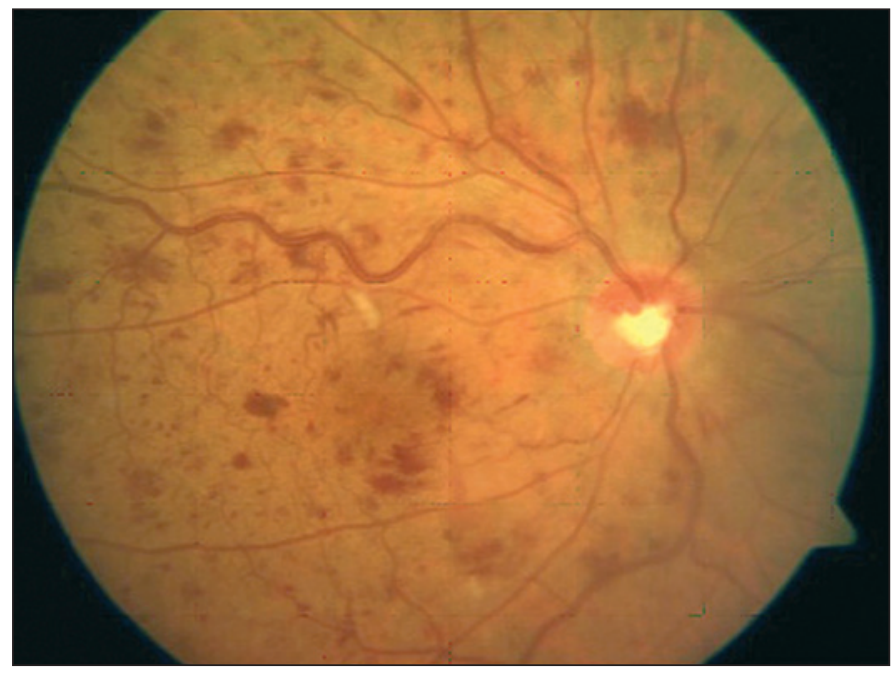

Figura 1 - Trombose de veia central da retina no olho direito
20/20 e J1 em ambos os olhos. O exame biomicroscópico, o de motricidade ocular extrínseca e os reflexos oculares mostravam-se sem alterações. A pressão intra-ocular foi de $14 \mathrm{mmHg}$, em razão do uso de cloridrato de betaxolol duas vezes ao dia em ambos os olhos. O exame fundoscópico evidenciou o nervo óptico com uma escavação aumentada em ambos os olhos, sem outras alterações.

Após dois meses, a paciente procurou atendimento queixando-se da diminuição súbita da acuidade visual no olho direito. $\mathrm{O}$ exame de acuidade visual apresentou movimentos de mãos e o exame fundoscópico constatou hemorragia vítrea. Com a absorção da hemorragia vítrea, quinze dias depois foi possível o exame da retina e constatar a forma não isquêmica de trombose da veia central. Três semanas após a baixa visual no olho direito, foram observadas, no olho contralateral, hemorragias retinianas profundas na arcada temporal superior (Figuras 1 e 2).

Exames colhidos durante o quadro agudo: contagem de plaquetas, hemograma, testes de coagulação, tempo de sangramento, proteína $\mathrm{S}$, proteína $\mathrm{C}$ e antitrombina sem alterações. Dosagem de homocisteína dentro da faixa de normalidade, ausência de mutação do fator $\mathrm{V}$ de Leiden e protrombina, ausência de anticoagulante lúpico. A velocidade de hemossedimentação (VHS) foi de $40 \mathrm{~mm}$.

Devido a esses achados e à ausência de outros fatores de risco, optou-se por interromper as medicações em uso. A paciente apresentou então uma melhora gradativa da visão. Atualmente sua melhor acuidade visual corrigida é de 20/40 e 20/20. A fundoscopia, após dez meses, apresenta, no olho direito, colaterais e engurgitamento venoso.

\section{DISCUSSÃO}

A oclusão de veia central da retina (OVCR) é tipicamente vista em pacientes com mais de 65 anos. Em algumas situações

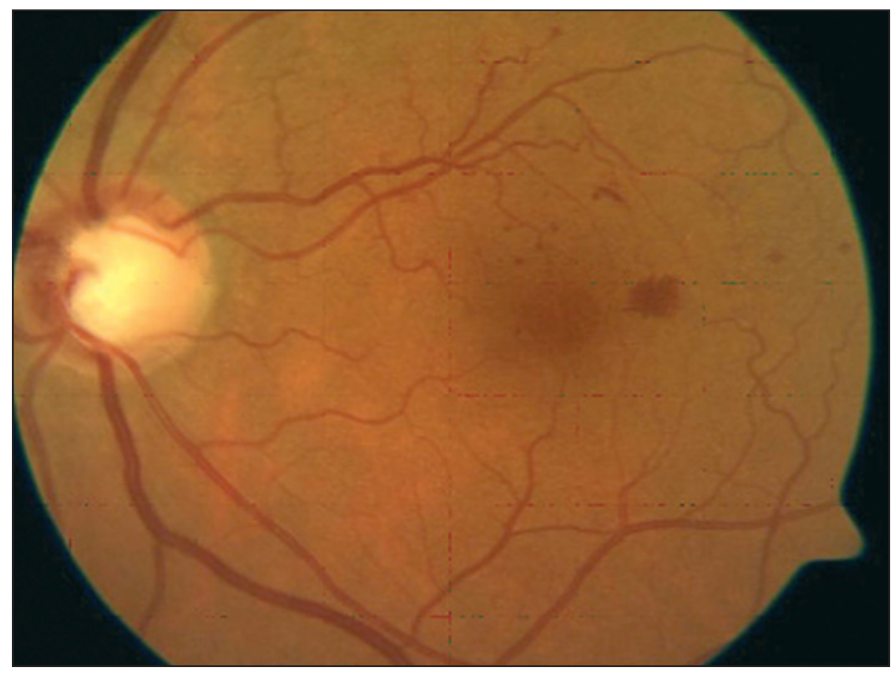

Figura 2 - Hemorragias retinianas no olho esquerdo 
temos uma disfunção endotelial com inflamação, edema e rotura vascular secundária ${ }^{(18)}$; em outras temos a formação do trombo ao nível da lâmina cribiforme ${ }^{(19)}$. O grande estudo "Eye Disease Case-Control Study" encontrou uma série de fatores de risco para a OVCR; os principais sendo hipertensão arterial sistêmica, diabetes mellitus e glaucoma primário de ângulo aberto.

Indivíduos jovens apresentaram maior associação com estados pró-trombóticos decorrentes de trombofilias congênitas ou adquiridas, por exemplo: deficiência de proteína $\mathrm{S}$, deficiência de proteína $\mathrm{C}$, resistência à proteína $\mathrm{C}$ ativada, presença do fator $\mathrm{V}$ de Leiden, presença de anticorpo antifosfolípide e níveis anormais de fibrinogênio ${ }^{(20-21)}$. Níveis elevados de homocisteína e estados de hiperviscosidade também apresentaram associação. Não há indicação de investigação laboratorial nos pacientes com mais de 60 anos, com fatores de risco sistêmicos conhecidos ${ }^{(22)}$. No caso acima relatado o único fator de risco apresentado pela paciente, além da idade, foi a presença do glaucoma.

Os pacientes com hepatite $\mathrm{C}$ podem apresentar oclusões vasculares retinianas em decorrência de patologias sistêmicas associadas, e trombofilias congênitas ou adquiridas, em decorrência da doença per se ou de seu tratamento. Tanto o vírus da hepatite $\mathrm{C}$ como o INF $\alpha$ induzem à formação de grande variedade de anticorpos trombogênicos, incluindo crioglobulinas, fator reumatóide, fator antimúsculo liso, antimicrossoma de fígado-rim, anticardiolipina e antifosfolípide (positivo em $22 \%$ dos pacientes com hepatite C), o que predispõe os pacientes portadores do vírus e/ou em tratamento com INF $\alpha$ ao desenvolvimento de trombose de veia retiniana ${ }^{(17)}$.

A incidência de retinopatia associada ao uso de INF $\alpha$ varia de 18 a $86 \%$ em pacientes orientais; e em pacientes nãoorientais, com hepatite $\mathrm{C}$ crônica tratada com INFo e ribavirina, chega a $42 \%^{(14,23)}$. A fisiopatologia da retinopatia associada ao uso de INF $\alpha$ não é ainda bem compreendida. Pressupõe-se que o INF $\alpha$ cause deposição de imunocomplexos na microcirculação capilar, o que provocaria isquemia retiniana, hemorragias e infartos da camada de fibras nervosas. Altos níveis de fator C5a ativados do complemento podem levar a microinfartos decorrentes da formação de microêmbolos vasculares $^{(13,24)}$. Esses êmbolos podem envolver raramente vasos de grande calibre e causar oclusões arteriais ${ }^{(16)}$. O tratamento com o interferon também está associado a risco aumentado de diabetes e hipertrigliceridemia, e pode ser um fator de risco adicional ao estado de hipercoagulabilidade ${ }^{(25)}$.

Muita discussão faz-se sobre a necessidade de exames rotineiros em todos os pacientes em uso destas medicações ${ }^{(15,26)} \mathrm{e}$ a necessidade de descontinuação aos primeiros sinais da retinopatia ${ }^{(14)}$. Na população geral, as lesões têm incidência baixa, são na sua maioria assintomáticas e desaparecem sem deixar seqüelas em uma grande porcentagem dos pacientes, mesmo com a manutenção do tratamento ${ }^{(14-15)}$. Todavia, em um pequeno grupo de indivíduos as complicações decorrentes da isquemia podem levar a morbidades mais graves, levando alguns estudos a sugerirem o "screening" e acompanhamento regu$\operatorname{lar}^{(13,27)}$. Outros estudos sugerem exames fundoscópicos antes do início do tratamento, e até contra-indicam o uso do interferon na presença de vasculopatia retiniana prévia (em específico a retinopatia diabética), além de acompanhamento trimestral daqueles que iniciarão o tratamento ${ }^{(7)}$.

Desse modo, o caso descrito é bastante ilustrativo, pois expõe em um dos olhos o quadro mais freqüente da retinopatia associada ao uso de interferon, i.e. algumas hemorragias em pólo posterior, e no olho contralateral uma apresentação muito mais atípica, uma trombose de veia central da retina. $\mathrm{O}$ aparecimento destes sinais teve surgimento mais tardio na paciente deste relato, em comparação com a literatura, cuja média de aparecimento foi de 4 meses após o início da medicação ${ }^{(14)}$.

Outro aspecto interessante a respeito deste caso é a ausência de quaisquer fatores de risco para o desenvolvimento das formas graves da retinopatia pelo interferon: doença vascular retiniana (retinopatia diabética e hipertensiva) ${ }^{(7)}$; doenças sistêmicas como diabetes, hipertensão e anemia ${ }^{(7,11,16,28)}$; dose inicial de interferon ${ }^{(7)}$ e a freqüência em que eram feitos os exames (pois as hemorragias e os exsudatos são fugazes, desaparecendo em poucas semanas) ${ }^{(14)}$. Não foram comprovados como fatores de risco os seguintes aspectos: duração do tratamento $^{(14)}$, alterações laboratoriais de hemograma, contagem de plaquetas e leucócitos ${ }^{(11,14)}$.

A incidência de retinopatia associada ao interferon talvez seja mais freqüente do que vemos na prática, justamente por não haver acompanhamento de rotina destes pacientes. Entretanto em sua grande maioria elas são assintomáticas e evoluem com melhora espontânea, não necessitando de intervenção ou interrupção do tratamento. Devemos buscar estudos que valorizem a procura pelos fatores de risco para as formas mais graves, para assim alertar o paciente, seu gastroenterologista e o oftalmologista.

\section{ABSTRACT}

Interferon and ribavirin are medications widely used in the treatment of some systemic diseases, mainly hepatitis C. Ribavirin when associated with interferon increases the rate of success of this treatment. There are about 170 million patients with chronic hepatitis $\mathrm{C}$ in the world, many in use of these medications. The classic associated retinopathy is described as cotton wool exudates and hemorrhages. Since the first reports, several different ocular disturbances were described in association with interferon. The present case shows a patient whose right eye presented with central retinal vein occlusion and whose left eye presented the typical findings of hemorrhages; prompt resolution after the medications were discontinued.

Keywords: Hepatitis C; Ribavirin/adverse effects; Interferon/ adverse effects; Retinopathy; Retinal vein occlusion; Case reports [publication type] 


\section{REFERÊNCIAS}

1. Alter MJ. Epidemiology of hepatitis C. Hepatology. 1997;26(3 Suppl):62S-65S.

2. Hepatitis C: global prevalence. Wkly Epidemiol Rec. 1997;72(46):341-4.

3. Poynard T, Marcellin P, Lee SS, Niederau C, Minuk GS, Ideo G, et al. Randomised trial of interferon alpha2b plus ribavirin for 48 weeks or for 24 weeks versus interferon alpha2b plus placebo for 48 weeks for treatment of chronic infection with hepatitis C virus. Lancet. 1998;352(9138):1426-32.

4. McHutchison JG, Gordon SC, Schiff ER, Shiffman ML, Lee WM, Rustgi VK, et al. Interferon alfa- $2 \mathrm{~b}$ alone or in combination with ribavirin as initial treatment for chronic hepatitis C. Hepatitis Interventional Therapy Group. N Engl J Med. 1998;339(21):1485-92.

5. Brok J, Gluud LL, Gluud C. Effects of adding ribavirin to interferon to treat chronic hepatitis $\mathrm{C}$ infection: a systematic review and meta-analysis of randomized trials. Arch Intern Med. 2005;165(19):2206-12.

6. Hayasaka S, Nagaki Y, Matsumoto M, Sato S. Interferon associated retinopathy. Br J Ophthalmol. 1998;82(3):323-5.

7. Patterson JL, Fernandez-Larsson R. Molecular mechanisms of action of ribavirin. Rev Infect Dis. 1990;12(6):1332-46.

8. Ning Q, Brown D, Parodo J, Cattral M, Gorczynski R, Cole E, et al. Ribavirin inhibits viral-induced macrophage production of TNF, IL-1, the procoagulant fg12 prothrombinase and preserves Th1 cytokine production but inhibits Th2 cytokine response. J Immunol. 1998;160(7):3487-93.

9. Borden EC, Parkinson D. A perspective on the clinical effectiveness and tolerance of interferon- alpha. Semin Oncol. 1998;25(1 Suppl 1):3-8.

10. Kawano T, Shigehira M, Uto H, Nakama T, Kato J, Hayashi K, et al. Retinal complications during interferon therapy for chronic hepatitis C. Am J Gastroenterol. 1996;91(2):309-13.

11. Chambers RB, Downie A, Foote B, Davidorf FH. Interferon alfa-associated retinopathy. J Am Osteopath Assoc. 1997;97(1):43-5.

12. Guyer DR, Tiedeman J, Yannuzzi LA, Slakter JS, Parke D, Kelley J, et al. Interferon-associated retinopathy. Arch Ophthalmol. 1995;111(3):350-6.

13. Jain K, Lam WC, Waheeb S, Thai Q, Heathcote J. Retinopathy in chronic hepatitis $\mathrm{C}$ patients during interferon treatment with ribavirin. $\mathrm{Br} \mathrm{J}$ Ophthalmol. 2001;85(10):1171-3

14. Kiratli H, Irkec M. Presumed interferon-associated bilateral macular arterial branch obstruction. Eye. 2000;14(Pt 6):920-2.
15. Sugano S, Suzuki T, Watanabe M, Ohe K, Ishii K, Okajima T. Retinal complications and plasma C5a levels during interferon alpha therapy for chronic hepatitis C. Am J Gastroenterol. 1998;93(12):2441-4.

16. Nadir A, Amin A, Chalisa N, van Thiel DH. Retinal vein thrombosis associated with chronic hepatitis C: a case series and review of literature. J Viral Hepat. 2000;7(6):466-70

17. Cuthbertson FM, Davies M, McKibbin M. Is screening for interferon retinopathy in hepatitis C justified? Br J Ophthalmol. 2004;88(12):1518-20.

18. Rubio JE Jr, Charles S. Interferon-associated combined branch retinal artery and central retinal vein obstruction. Retina. 2003;23(4):546-8.

19. Chuman T, Nao-i N, Sawada A, Kawano T, Shigehira M. [Interferon-induced retinal changes]. Nippon Ganka Gakkai Zasshi. 1994;98(6):616-21. Japanese.

20. Willson RA. Visual side effects of pegylated interferon during therapy for chronic hepatitis C infection. J Clin Gastroenterol. 2004;38(8):717-22.

21. Schulman JA, Liang C, Kooragayala LM, King J. Posterior segment complications in patients with hepatitis $\mathrm{C}$ treated with interferon and ribavirin. Ophthalmology. 2003;110(2):437-42.

22. Davis GL, Esterbaun-Mur R, Rustigi V, Hoefs J, Gordon SC, Trepo C, et al. Interferon alpha- $2 \mathrm{~b}$ alone or in combination with ribavirin for the treatment of relapse of chronic hepatitis C. International Hepatitis Interventional Therapy Group. N Engl J Med. 1998;339(21):1493-9.

23. Risk factors for central retinal vein occlusion. The Eye Disease Case-Control Study Group. Arch Ophthalmol. 1996;114(5):545-54.

24. Green W, Chan CC, Hutchins GM, Terry JM. Central retinal vein occlusion: a prospective histopathologic study of 29 eyes in 28 cases. Trans Am Ophthalmol Soc. 1981;79:371-422.

25. Williamson TH, Rumley A, Lowe GD. Blood viscosity, coagulation, and activated protein $\mathrm{C}$ resistance in central retinal vein occlusion: a population controlled study. Br J Ophthalmol. 1996;80(3):203-8.

26. Gottlieb JL, Blice JP, Mestichelli B, Konkle BA, Benson WE. Activated protein $\mathrm{C}$ resistance, factor $\mathrm{V}$ Leiden, and central retinal vein occlusion in young adults. Arch Ophthalmol. 1998;116(5):577-9.

27. Bearelly S, Fekrat S. Controversy in the management of retinal venous occlusive disease. Int Ophthalmol Clin. 2004;44(4):85-102.

28. Fabris P, Betterle C, Greggio NA, Zanchetta R, Bosi E, Biasin MR, de Lalla F. Insulin-dependent diabetes mellitus during alpha-interferon therapy for chronic viral hepatitis. J Hepatol. 1998;28(3):514-7.

\section{Congresso da SOTRIM Sociedade de Oftalmologia do Triângulo Mineiro}

